\title{
Circumstances of discovery of phaeochromocytoma: a retrospective study of 41 consecutive patients
}

\author{
Jean-Philippe Baguet, Laure Hammer, Tânia Longo Mazzuco ${ }^{1}$, Olivier Chabre ${ }^{1}$, Jean-Michel Mallion, \\ Nathalie Sturm ${ }^{2}$ and Philippe Chaffanjon ${ }^{3}$ \\ Department of Cardiology and Hypertension, ${ }^{1}$ Department of Endocrinology, ${ }^{2}$ Department of Pathology and ${ }^{3}$ Department of Thoracic and General Surgery, \\ Grenoble University Hospital, 38043 Grenoble Cedex 09, France
}

(Correspondence should be addressed to J-P Baguet; Email: JPBaguet@chu-grenoble.fr)

\begin{abstract}
Objective: Phaeochromocytoma is a rare tumour of the chromaffin cells, the diagnosis of which is based on an assay of metanephrines and treatment is surgical excision of the tumour. It is usually discovered due to a rich and varied symptomatology or classic paroxysmal hypertension. The main purpose of this study was to specify the exact circumstances of discovery of the phaeochromocytomas operated on in our university hospital between 1990 and 2002.

Design and methods: Forty-one consecutive and complete case reports of patients who had surgery for phaeochromocytoma were analysed retrospectively. This series includes 10 patients with a genetic disorder predisposing to phaeochromocytoma.

Results: The association of headaches and palpitations with sweating was found in only $24 \%$ of cases $(10 / 41)$. Blood pressure anomalies led to the discovery of phaeochromocytoma in only $51 \%$ of cases $(21 / 41)$ and $59 \%(24 / 41)$ of all the patients suffered from hypertension. In almost half the cases $(20 / 41)$, the tumour was discovered by an imaging method (ultrasonography, CT scan or MRI) which had been performed for reasons unrelated to a blood pressure abnormality.

Conclusions: Phaeochromocytoma, the symptoms of which are not very specific and during which hypertension is present in only half the patients, is a disease that remains rare. Its incidence could be increasing because of changes in the method of detection. Indeed, in our study, different imaging techniques led to its incidental discovery in half of the cases.
\end{abstract}

European Journal of Endocrinology 150 681-686

\section{Introduction}

Phaeochromocytoma is a rare tumour (approximately one hypertensive subject in 1000) of the chromaffin cells which secrete catecholamines and/or their metabolites (1). In $90 \%$ of cases it arises from the adrenal medulla but it may also be revealed in chromaffin cells of sympathetic paraganglia located throughout the body, from the neck to the bladder. Its prevalence is identical in both sexes and its incidence maximal between the ages of 40 and 60. It varies in size but rarely exceeds $60 \mathrm{~mm}$ along the long axis. It is bilateral in $10 \%$ of cases $(20 \%$ in children) and usually $(90 \%)$ benign. However, regardless of its histological aspect, the tumour cannot be assumed to be benign at the time of diagnosis. Extra-adrenal phaeochromocytomas, also called paragangliomas, are more often malignant (more than 30\%) (2). In 20-30\% of the cases, phaeochromocytoma is part of a genetic disease with autosomal dominant inheritance: multiple endocrine neoplasia (MEN) type 2, Von Hippel-Lindau's disease (VHL), or neurofibromatosis type 1 (NF1, Von Recklinghausen's disease) $(3,4)$.

Hormonal diagnosis is based on the measurements of urinary or plasma catecholamines or the catecholamine metabolites metanephrines. The treatment of choice is surgical excision of the tumour. Although the prognosis for patients who have surgery for benign phaeochromocytoma is identical to that of healthy patients, the prognosis in cases of malignant phaeochromocytoma is poor. Hypertension, the classic condition leading to the discovery of phaeochromocytoma, may be cured after surgical excision but persists in one-third of the patients who undergo surgery.

The purpose of this study was to determine the circumstances of discovery of phaeochromocytoma in 41 consecutive cases operated at the Grenoble University Hospital between 1990 and 2002. 


\section{Subjects and methods}

We have retrospectively studied the files of all patients who underwent surgery for phaeochromocytoma at Grenoble University Hospital between 1990 and 2002. The cases were identified from the files of the Department of Pathology and included patients followed in the departments of cardiology, endocrinology, internal medicine and other departments of our hospital. The completeness of the case report forms drawn up by a senior physician was verified prior to their inclusion. For the purposes of this study, medical records on the first consultation were analysed to establish how diagnosis was made. Detailed personal and family history, physical examination, biochemical analysis and imaging were also reviewed at the time the tumour was detected. Two incomplete case report forms (exact circumstances of discovery of phaeochromocytoma were missing) were not included and only 41 of 43 cases were analysed. Clinical blood pressure (BP) measurements were recorded on therapy. The following assay methods were used: photometry for total urinary metanephrines and urinary vanillylmandelic acid (VMA); fluorimetry for total urinary catecholamines; and HPLC for plasma metadrenaline, normetadrenaline and catecholamines (5). Urinary analyses were performed at Grenoble University Hospital and plasma analysis at Lyon University Hospital. Statistical analysis was performed using SPSS software (SPSS Inc., Chicago, IL, USA). The mean and standard deviation were calculated for each numerical variable.

\section{Results}

The characteristics of the 41 patients selected are shown in Table 1. They were aged between 14 and 79. Eleven patients had a family history of hypertension $(27 \%)$ and two suffered from refractory hypertension (5\%). Ambulatory BP monitoring (ABPM) was performed in 12 patients $(29 \%)$ with a mean daytime BP of $142 \pm 21$ for systolic BP (SBP) and 92 $\pm 15 \mathrm{mmHg}$ for diastolic BP (DBP). A hypertensive peak, defined for both clinical and ambulatory measurements as a sudden elevation in SBP by more than $50 \%$ of the

Table 1 Characteristics of the 41 patients who had surgery for a phaeochromocytoma.

\begin{tabular}{lc}
\hline Variables & Mean \pm SD \\
\hline Gender (men/women) & $16 / 25$ \\
Age (years) & $46 \pm 16$ \\
Body mass index $\left(\mathrm{kg} / \mathrm{m}^{2}\right)$ & $23 \pm 4$ \\
Cardiovascular heredity (yes/no) & $11 / 30$ \\
Clinical SBP (mmHg) & $142 \pm 21$ \\
Clinical DBP (mmHg) & $87 \pm 14$ \\
Clinical heart rate (b.p.m.) & $76 \pm 14$ \\
Glucose level (mmol/l) & $6.9 \pm 3.0$ \\
\hline
\end{tabular}

preceding measurement, was detected clinically in $49 \%$ of the patients and in $67 \%$ of the subjects when using ABPM. Nineteen patients (46\%) were being treated for hypertension at the time of phaeochromocytoma diagnosis, and had suffered from hypertension for an average of $54 \pm 45$ months (maximum $=10$ years). In the treated patients, the average period of anti-hypertensive drugs was $35 \pm 35$ months and the average number of anti-hypertensive medication was $2.2 \pm 0.4$. Seven patients $(17 \%)$ had NF1; two related patients $(5 \%)$ suffered from MEN type $2 \mathrm{~A}$ with a mutation in the RET gene at codon 634. One patient $(2 \%)$ with a bilateral phaeochromocytoma proved to carry a missense germinal neomutation in the gene for VHL.

Table 2 reports the clinical symptoms and signs of patients before surgery for phaeochromocytoma. The different ways of detecting the tumours are given in Table 3. BP anomalies led to the discovery of phaeochromocytoma in $51 \%$ of cases $(21 / 41)$ : paroxysmal, symptomatic, refractory or gravidic hypertension. Hypertension, defined by a clinical SBP $\geq 140 \mathrm{mmHg}$ and/or a clinical $\mathrm{DBP} \geq 90 \mathrm{mmHg}$ and/or current anti-hypertensive treatment (6), was found in 59\% $(24 / 41)$ of the patients. Hypertension was grade 1 (SBP between 140 and $159 \mathrm{mmHg}$ and/or DBP between 90 and $99 \mathrm{mmHg}$ ) in nine of the $24(37.5 \%)$ hypertensive subjects, grade 2 (SBP between 160 and $179 \mathrm{mmHg}$ and/or DBP between 100 and $109 \mathrm{mmHg})$ in nine cases $(37.5 \%)$ and grade 3 (SBP > $180 \mathrm{mmHg}$ and/or DBP > $110 \mathrm{mmHg}$ ) in six of the $24(25 \%)$ patients. Among the 19 treated patients, $15(79 \%)$ had a persistent hypertension. Orthostatic hypotension was defined as a fall in BP $1 \mathrm{~min}$ after standing, by more than $20 \mathrm{mmHg}$ for the SBP and more than $10 \mathrm{mmHg}$ for the DBP. Twelve subjects $(29 \%)$ did not display either signs of the triad or orthostatic hypotension. Of the patients whose phaeochromocytoma was discovered during an imaging examination, 35\% were hypertensive.

The results of preoperative hormonal assays are given in Table 4. All of the patients had at least one abnormal hormonal assay. Only two patients out of

Table 2 Symptoms and clinical signs of the 41 patients before surgery for phaeochromocytoma.

\begin{tabular}{lc}
\hline Variables & Percentage (yes/no) \\
\hline Headaches & $49(20 / 21)$ \\
Palpitations & $46(19 / 22)$ \\
Sweating & $49(20 / 21)$ \\
Headaches + palpitations + sweating & $24(10 / 31)$ \\
Absence of symptoms & $29(12 / 41)$ \\
Hypertension & $59(24 / 41)$ \\
Orthostatic hypotension & $15(6 / 35)$ \\
Clinical BP peak & $49(20 / 21)$ \\
ABPM peak & $67(8 / 4)$ \\
Acute pulmonary oedema & $2(1 / 40)$ \\
\hline
\end{tabular}


Table 3 Circumstances of discovery of 41 cases before surgery for phaeochromocytoma.

\begin{tabular}{lcc} 
& Percentage (\%) & Presence (/41) \\
\hline Hypertension & 51 & 21 \\
Paroxysmal HT & 11 \\
Aetiological report of HT & 9 \\
Gravidic HT & 1 \\
Imaging for pain examination & 29 & 12 \\
Abdominal pain & 7 \\
Nephretic colic & 1 \\
Urinary infection & 2 \\
Hepatic colic & 1 \\
Lumbar pain & 1 \\
Imaging for various pathologies & 20 & 8 \\
Uterine fibroma & 1 \\
Elevated transaminases & 1 \\
Bone metastases & 1 \\
Hypereosinophilia & 1 \\
Pregnancy monitoring & 1 \\
MEN 2A* & 2 \\
Von Recklinghausen's disease* & 1
\end{tabular}

HT, hypertension.

*After finding elevated level of metanephrines.

$29(7 \%)$ had a normal level of urinary metanephrines, six $(21 \%)$ had up to twice the normal level and 21 (72\%) had more than twice the normal level. Plasma metanephrines were normal in $21 \%$ of cases (4/19) and urinary VMA in $12 \%$ of patients (3/24). Fiftyfour per cent $(22 / 41)$ of patients had fasting glucose levels higher than $6.0 \mathrm{mmol} / \mathrm{l}$.

Thirty-nine patients (95\%) had at least one adrenal tumour, in one case associated with a coeliac paraganglioma and in another case with a vertebral neurofibroma. Twenty-one of the adrenal tumours were on the right side, 15 on the left and three were bilateral. The other two subjects suffered from an inter-aortocaval paraganglioma. Metastases were detected at the time of diagnosis in two of the 41 patients. All of the patients were given a preoperative computerized tomography (CT) scan or magnetic resonance imaging (MRI). Total body scan by metaiodobenzylguanidine was performed in 15 patients. This exam was concordant with surgical findings in 14 cases (one bilateral adrenal fixation detected by this method but a unilateral tumour found during the surgery). From 1990 to
1996, the surgical approach was a laparotomy in all cases $(21 / 21)$, whereas from 1997 to 2002, 11 of the 20 patients $(55 \%)$ underwent a laparoscopic approach. Six $(15 \%)$ of the patients encountered intra- or peri-operative complications: eventration, peritonitis, sub-hepatic haematoma, catheter subclavian thrombophlebitis, duodenal ulcer bleeding and acute pulmonary oedema. There was no mortality.

Table 5 shows the comparison of patients' characteristics and tumour data according to the circumstances of detection of the phaeochromocytoma. When the tumour was detected because of hypertension abnormality, the plasma normetadrenaline level was higher $(P=0.006)$ and there was a tendency towards higher SBP $(P=0.123)$, urinary VMA level $(P=0.158)$ and tumour weight $(P=0.054)$.

\section{Discussion}

\section{Hypertension and phaeochromocytoma}

Phaeochromocytoma is a disease which is often evoked but rarely found. The most characteristic clinical sign of this pathology and the one most frequently reported in the literature (in almost $90 \%$ of cases) is hypertension, which may be only paroxysmal but is usually permanent, stable or with paroxysms, sometimes even malignant (7-9). This hypertension, which shows extreme variation between levels, usually resists antihypertensive drugs apart from $\alpha$-blockers.

In our study, hypertension led to the discovery of phaeochromocytoma in only $51 \%$ of patients, and in half these cases because of its evocative paroxysmal character. When the reason for discovering the phaeochromocytoma was permanent hypertension, it was symptomatic, severe, resisting treatment, or gravidic. We found only six patients (15\%) with severe (grade 3) hypertension. This low prevalence is explained by the use of anti-hypertensive drugs in 19 of the 24 hypertensives and the high prevalence of paroxysmal hypertension with only 59\% suffering from permanent clinical hypertension before surgery. Moreover, we found a very high prevalence of uncontrolled hypertension in spite of an average number of anti-hypertensive medication of 2.2. This is well

Table 4 Preoperative hormonal data and tumour weight for the 41 patients suffering from phaeochromocytoma.

\begin{tabular}{|c|c|c|c|c|}
\hline Variables & Mean \pm SD & $n$ & Normal values & Median (range) \\
\hline Urinary metanephrines ( $\mu \mathrm{mol} / 24 \mathrm{~h})$ & $36.5 \pm 32.4$ & 29 & $<4.2$ & $31.6(2.8-99.3)$ \\
\hline Urinary VMA $(\mu \mathrm{mol} / 24 \mathrm{~h})$ & $79.8 \pm 63.7$ & 24 & $<30.3$ & $55.8(26.1-244)$ \\
\hline Plasma metadrenaline (pg/ml) & $5852 \pm 7685$ & 19 & $<1500$ & $1818(178-25824)$ \\
\hline Plasma normetadrenaline (pg/ml) & $23529 \pm 30499$ & 19 & $<4000$ & $5069(811-97638)$ \\
\hline Urinary catecholamines (nmol/24 h) & $8058 \pm 7965$ & 24 & $<1200$ & $4945(621-27061)$ \\
\hline Plasma catecholamines (pg/ml) & 85 & 1 & $<40$ & $85(85)$ \\
\hline Tumour weight $(\mathrm{g})$ & $103 \pm 148$ & & & $37(4.9-661)$ \\
\hline
\end{tabular}


Table 5 Comparison of data according to the circumstances of discovery of the 41 operated cases of phaeochromocytoma.

\begin{tabular}{lcc}
\hline Variables & BP abnormality $(n=21)$ & Other circumstance $(n=20)$ \\
\hline Gender (men/women) & $12 / 9$ & $4 / 16$ \\
Age (years) & $45 \pm 19$ & $47 \pm 13$ \\
Clinical SBP (mmHg) & $147 \pm 22$ & $137 \pm 18$ \\
Clinical DBP (mmHg) & $89 \pm 17$ & $85 \pm 9$ \\
Clinical heart rate (b.p.m.) & $78 \pm 16$ & $74 \pm 11$ \\
Glucose level (mmol/l) & $6.8 \pm 3.1$ & $7 / 21$ \\
Headaches+palpitations + sweating $(\mathrm{Y} / \mathrm{N})$ & $17 / 21$ & $3.9 \pm 3.0$ \\
High BP level $(\mathrm{Y} / \mathrm{N})$ & $15 / 21$ & $3 / 20$ \\
Treated hypertension $(\mathrm{Y} / \mathrm{N})$ & $43.1 \pm 32.9(n=14)$ & $4 / 20$ \\
Urinary metanephrines $(\mu \mathrm{mol} / 24 \mathrm{~h})$ & $98.4 \pm 73.2(n=12)$ & $30.3 \pm 31.9(n=15)$ \\
Urinary VMA $(\mu \mathrm{mol} / 24 \mathrm{~h})$ & $7264 \pm 8320(n=7)$ & $61.2 \pm 48.6(n=12)$ \\
Plasma metadrenaline $(\mathrm{pg} / \mathrm{ml})$ & $44470 \pm 35892(n=8)$ & $4954 \pm 7523(n=11)$ \\
Plasma normetadrenaline $(\mathrm{pg} / \mathrm{ml})$ & $58 \pm 60$ & $150 \pm 195$ \\
Tumour weight $(\mathrm{g})$ & & \\
\hline
\end{tabular}

$\mathrm{Y}$, yes; N, no.

described in secondary hypertension, especially in phaeochromocytoma. Hypertension is far from constant during phaeochromocytoma and its absence should not be a reason for rejecting a clinical diagnosis. Asymptomatic or slightly symptomatic characteristics and absence of hypertension in a patient suffering from phaeochromocytoma is usually explained by a tumour secreting very little catecholamines and with a discontinuous pattern. Thus, in our study, we found a significant lower plasma normetadrenaline level in patients for which the discovery of the tumour was not a BP abnormality. The ABPM performed over $24 \mathrm{~h}$ at the time of the metanephrine assay usually revealed hypertensive peaks more frequently than did clinical examination and is therefore more useful in revealing a paroxysmal rise in arterial pressure.

Apart from hypertension, clinical examination is usually normal in patients suffering from phaeochromocytoma. Some authors have found that hypertension linked to phaeochromocytoma is often associated with orthostatic hypotension explained by the downregulation of $\alpha$-adrenergic receptors $(10,11)$. This hypotension was only detected in six of our 41 patients. This low rate $(15 \%)$ of orthostatic hypotension in our study could also be due to moderate secretion of catecholamines.

\section{Other symptoms and clinical signs}

Phaeochromocytoma must be evoked when intense pulsing headache, profuse sweating and palpitations are associated (12). The sensitivity and specificity of this triad are high (13) but it may be present during an attack of hypertension in the absence of a phaeochromocytoma or in perimenopausal women. Its presence seems to be more important in detecting phaeochromocytoma when it is linked to orthostatic hypotension. In our study, each element of this triad was only present in half the patients and only $24 \%$ displayed all three symptoms. These findings are also reported in other studies $(8,9)$.

In the present study, only two patients displayed acute pulmonary oedema but this did not lead to discovery of the tumour. This complication can be due to a very high BP level or can be secondary to adrenergic myocarditis with, on echocardiography, frequent association of left ventricular hypertrophy and ventricular dilation (14).

Historically, genetic factors were believed to be implicated in only $10 \%$ of phaeochromocytoma, but recent data suggest that germline mutations may be detected in approximately 25\% of unselected cases. The most frequent causes of genetic predisposition for phaeochromocytoma are VHL, MEN type 2, the newly delineated phaeochromocytoma-paraganglioma syndrome and, less commonly, NF1 (3). In our study, phaeochromocytoma had been diagnosed by systematic screening with metanephrine in two patients previously known to be affected by MEN $2 \mathrm{~A}$ and in one patient suffering from NF1. In the other six patients with NF1, diagnosis of phaeochromocytoma was made only when the patients became hypertensive. Similarly, diagnosis of VHL was made after paroxysmal hypertension led to the diagnosis of a bilateral phaeochromocytoma in a 14-year-old boy with no other manifestation of VHL and no family history. Genetic analysis of leukocyte DNA identified a germinal missense neomutation of the VHL gene consistent with VHL type 2C.

\section{Biological diagnosis}

In phaeochromocytoma, there is an intermittent or permanent increase in the excretion of catecholamines and their metabolites, metanephrines. These compounds can be assayed in plasma or urine. Phaeochromocytomas secreting dopamine are exceptional. 
A urinary metanephrine assay is currently the standard examination for phaeochromocytoma diagnosis. Its sensitivity and specificity approach $100 \%$ when HPLC is used $(15,16)$. This was found in our study where $93 \%$ of urinary metanephrines assays were pathological.

In addition, fasting hyperglycaemia is common in phaeochromocytoma because of insulin resistance due to desensitization of $\beta$-adrenergic receptors and inhibition of insulin secretion by the activation of $\alpha$-adrenergic receptors. Thus, half the patients in our study had a blood glucose level higher than $6.0 \mathrm{mmol} / \mathrm{l}$.

\section{Role of imaging in diagnosis}

When an abnormally high level of excretion of metanephrines is recorded, the tumour must be located. CT scan or MRI detect one or more tumours in most cases, specify their anatomical relations and detect metastases. On the other hand, these same imaging techniques along with ultrasonography may find an abnormal mass which could be a phaeochromocytoma (17). Imaging is increasingly used in medical practice and for many indications. It is therefore not surprising, among the methods of detecting phaeochromocytoma, to find a high percentage of pathologies which led to abdominal, pelvic or retroperitoneal imaging being performed. Thus, in our study, $49 \%$ of phaeochromocytomas were discovered by ultrasound, CT scan or MRI. The reason for performing these examinations was initially a painful abdominal or lumbar syndrome. These data are very close to those published by Noshiro et al., who had found that of 46 patients suffering from phaeochromocytoma diagnosed between 1986 and 1995 , in 19 cases $(41 \%)$ the tumour was discovered quite accidentally by imaging prescribed for other causes (liver dysfunction, upper abdominal pain, health check, weight loss, lumbar pain, abdominal discomfort, and hepatomegaly) (9). In a study performed at the Mayo Clinic, only 15 of 150 patients with a phaeochromocytoma were discovered serendipitously by abdominal CT (18). In our series, the high prevalence of patients with phaeochromocytoma discovered by imaging might be due to the 'comprehensive' nature of this series which included all patients operated for phaeochromocytoma in our university hospital and not only patients explored in cardiological or endocrine units. It has also already been demonstrated that any adrenal mass must lead to a search for phaeochromocytoma, even in the absence of hypertension. Interestingly, in our study, tumour weight was three times higher in subjects with incidental discovery of phaeochromocytoma ( 150 vs $58 \mathrm{~g}, P=0.056$ ). This should be explained by the low rate of tumoral secretion despite the size of the phaeochromocytoma, and by the fact that the larger the tumour, the easier it is to detect using imaging.

\section{Conclusions}

In conclusion, phaeochromocytoma is a pathology that remains rare but its incidence could increase following changes in the methods used to detect it. Indeed, various imaging techniques led, in our study, to its incidental discovery in half the cases. Its symptomatology is not very specific and hypertension is present in only half the patients.

\section{Acknowledgements}

We thank Dr Patrice Faure and Dr Jean-Marie CottetEmard for the hormone analyses. This study was supported in part by PHRC grant AOMO2068 and by grants from INSERM and Ministère Délégué à la Recherche et des Neuvelles Technologies for the COMPETE Network.

\section{References}

1 Manger WM, Gifford RW Jr. Pheochromocytoma. Berlin: Springer, 1977.

2 O'Riordain DS, Young WF, Grant CS, Carney JA \& van Heerden JA. Clinical spectrum and outcome of functional extraadrenal paraganglioma. World Journal of Surgery 199620 916-921.

3 Maher ER \& Eng C. The pressure rises: update on the genetics of phaeochromocytoma. Human Molecular Genetics 200211 2347-2354.

4 Bryant J, Farmer J, Kessler LJ, Townsend RR \& Nathanson KL. Pheochromocytoma: the expanding genetic differential diagnosis. Journal of the National Cancer Institute 200395 1196-1204.

5 Mornex R, Peyrin L, Pagliari R \& Cottet-Emard JM. Measurement of plasma methoxyamines for the diagnosis of pheochromocytoma. Hormone Research $199136220-226$.

6 European Society of Hypertension-European Society of Cardiology guidelines for the management of arterial hypertension. Guidelines Committee. Journal of Hypertension $2003 \mathbf{2 1}$ 1011-1053.

7 Januszewicz W \& Wocial B. Clinical and biochemical aspects of pheochromocytoma. Report of 110 cases. Cardiology $1985 \mathbf{7 2}$ (Suppl 1) 131-136.

8 Goldstein RE, O'Neill JA Jr, Holcomb GW 3rd, Morgan WM 3rd, Neblett WW 3rd, Oates JA et al. Clinical experience over 48 years with pheochromocytoma. Annals of Surgery 1999229 755-766.

9 Noshiro T, Shimizu K, Watanabe T, Akama H, Shibukawa S, Miura $\mathrm{W}$ et al Changes in clinical features and long-term prognosis in patients with pheochromocytoma. American Journal of Hypertension 200013 35-43.

10 Smithwick RH, Greer NER \& Robertson CW. Pheochromocytoma: a discussion of symptoms, signs and procedures of diagnostic value. New England Journal of Medicine 1950242 252-257.

11 Streeten DH \& Anderson GH Jr. Mechanisms of orthostatic hypotension and tachycardia in patients with pheochromocytoma. American Journal of Hypertension 19969 760-769.

12 Thomas JE, Rooke JD \& Kvale WF. The neurologist's experience with pheochromocytoma. A review of 100 cases. JAMA 1966 $197754-758$.

13 Plouin PF, Degoulet P, Tugayé A, Ducrocq MB \& Ménard J. Screening for phaechromocytoma: in which hypertensive patients? A semiological study of 2585 patients, including 11 with phaeochromocytoma. La Nouvelle Presse Medicale 198110 869-872.

14 Imperato-McGinley J, Gautier TK, Zullo MA, Goldstein DS \& Vaughan ED. Reversibility of catecholamine induced dilated cardiomyopathy in a child with a pheochromocytoma. New England Journal of Medicine $1987316793-797$. 
15 Krstulovic AM. Investigations of catecholamine metabolism using high-performance liquid chromatography: analytical methodology and clinical applications. Journal of Chromatography $19822291-34$.

16 Graham PE, Smythe GA, Edwards GA \& Lazarus L. Laboratory diagnosis of phaeochromocytoma: which analytes should we measure? Annals of Clinical Biochemistry 199330 129-134.

17 Lockhart ME, Smith JK \& Kenney PJ. Imaging of adrenal masses. European Journal of Radiology $2002 \mathbf{4 1} 95-112$.
18 Kudva YC, Young WF Jr \& Thompson GB. Adrenal incidentaloma: an important component of the clinical presentation spectrum of benign sporadic adrenal pheochromocytoma. Endocrinologist $1999977-84$

Received 7 October 2003

Accepted 2 February 2004 